\title{
Fatores demográficos e comportamentais associados à obesidade abdominal em usuárias de centro de saúde de Belo Horizonte, Minas Gerais, Brasil
}

\section{Demographic and behavioral factors associated with abdominal obesity in women attending a health} care unit in Belo Horizonte, Minas Gerais, Brazil

Edílson Ornelas OLIVEIRA ${ }^{1}$

Gustavo VELÁSQUEZ-MELÉNDEZ1

Gilberto KAC²

\section{R E S U M O}

\section{Objetivo}

Identificar variáveis sócio-demográficas e comportamentais potencialmente associadas à obesidade abdominal, em mulheres usuárias de unidade básica de saúde.

\section{Métodos}

Foram investigadas 827 mulheres entre 12 e 65 anos, residentes na região Metropolitana de Belo Horizonte, Minas Gerais, Brasil, utilizando-se desenho transversal. A obesidade abdominal foi definida como valores de circunferência da cintura $\geq 88 \mathrm{~cm}$. A associação entre obesidade abdominal e as co-variáveis foi avaliada por meio da técnica de regressão logística não condicional, com modelos hierarquizados, que estimam os Odds Ratio e os respectivos intervalos de confiança de $95 \%$.

\section{Resultados}

As maiores prevalências de obesidade abdominal foram encontradas em mulheres $\geq 51$ anos $(63,3 \%)$ e em mulheres com $\geq 5$ partos $(53,8 \%)$. As seguintes variáveis permaneceram associadas à obesidade abdominal no modelo final: idade (Odds Ratio variando entre 2,53 e 27,64), escolaridade $<8$ anos (Odds Ratio $=1,49$, intervalo de confiança 95\%: 1,02 - 2,18), paridade 1 - 4 filhos (Odds Ratio=1,80, intervalo de confiança 95\%: 1,04 - 3,12), $\geq 5$ filhos (Odds Ratio=3,20, intervalo de confiança 95\%: 1,48 - 6,90) e atividade física leve no trabalho (Odds Ratio=1,63, intervalo de confiança 95\%: 1,01 - 2,64). As variáveis idade e índice de massa corporal também foram incluídas no modelo final para controlar confundimento.

\footnotetext{
1 Universidade Federal de Minas Gerais, Escola de Enfermagem, Departamento de Enfermagem Materno Infantil e Saúde Pública. Av. Alfredo Balena, 190, 30130-100, Belo Horizonte, MG, Brasil. Correspondência para/Correspondence to: G. VELÁSQUEZ-MELÉNDEZ. E-mail: <guveme@ufmg.br>.

2 Universidade Federal do Rio de Janeiro, Instituto de Nutrição Josué de Castro, Departamento de Nutrição Social e Aplicada. Rio de Janeiro, RJ, Brasil.
} 


\section{Conclusão}

Conclui-se que a escolaridade e a paridade são potenciais preditores para o desenvolvimento da obesidade abdominal no grupo estudado e devem ser levados em consideração em estudos sobre obesidade.

Termos de indexação: antropometria; escolaridade; fatores de risco; mulheres; obesidade; paridade.

\section{A B S T R A C T}

\section{Objective}

To identify sociodemographic and behavioral variables potentially associated with abdominal obesity in women attending a health care unit.

\section{Methods}

A total of 827 women aging from 12 to 65 years and living in the metropolitan area of Belo Horizonte, Brazil, were investigated using a cross-sectional design. Abdominal obesity was defined as waist circumference $\geq 88 \mathrm{~cm}$. The association between abdominal obesity and the co-variables was assessed by unconditional logistic regression. This technique estimates the odds ratio and the respective confidence intervals of 95\%.

\section{Results}

The highest prevalence of abdominal obesity was observed in women aging $\geq 51$ years (63.3\%) and women with $\geq 5$ deliveries (53.8\%). The following variables remained associated with abdominal obesity in the final model: age (Odds Ratio varied from 2.53 to 27.64), schooling $<8$ years (Odds Ratio=1.49, confidence intervals 95\%: 1.02 - 2.18), parity 1 - 4 deliveries (Odds Ratio=1.80, confidence intervals 95\%: $1.04-3.12$ ), $\geq 5$ deliveries (Odds Ratio=3.20, confidence intervals 95\%: 1.48 - 6.90) and light physical activity at work (Odds Ratio=1.63, confidence intervals 95\%: 1.01 - 2.64). Age and body mass index were also included in the final model to control for confounding.

\section{Conclusion}

Schooling and parity are important predictors of abdominal obesity in the studied group and studies on obesity should take them into account.

Indexing terms: anthropometry; educational status; risk factors; women; obesity; parity.

\section{N T R O D U Ç Ã O}

Estudos populacionais realizados no Brasil, nos últimos 25 anos, têm demonstrado significativos aumentos nas prevalências de sobrepeso e obesidade em todas as regiões do País, tanto em homens como em mulheres ${ }^{1}$. O consumo de dietas energeticamente mais densas, a redução no gasto energético e o sedentarismo têm sido levantados como hipóteses para aumentos significativos do sobrepeso/obesidade em várias populações².

Embora existam aparentes diferenças étnico-raciais ${ }^{3}$ na distribuição da gordura corporal, o acúmulo de gordura no abdômen constitui-se em fator de risco independente para doenças do coração, hipertensão arterial e alterações metabólicas, como intolerância à glicose ${ }^{4}$. Dessa forma, o diagnóstico de obesidade abdominal pode subsidiar a identificação de indivíduos sob risco de doenças crônicas, como inicialmente proposto por Han et al. ${ }^{5}$. Devido à sua alta correlação com a quantidade de tecido adiposo visceral, a circunferência da cintura é a medida antropométrica preferencialmente usada para estimar o acúmulo de gordura abdominal ${ }^{3,6}$.

No Brasil, vários estudos de base populacional têm mostrado que fatores sócio-demográficos e comportamentais estão associados ao aumento do peso corporal. Entretanto, ainda existem poucos estudos acerca do papel desses determinantes na distribuição da gordura corporal ${ }^{7-10}$. Em geral, os riscos de desenvolver obesidade abdominal aumentam com a idade, com a paridade e diminuem com a maior escolaridade 8,11,12. Adicionalmente, observa-se associação entre obesidade abdominal e menopausa, consumo de cigarros e álcool ${ }^{6}$. 
Frente às repercussões negativas da obesidade e sua relação com o desenvolvimento de doenças crônicas não transmissíveis (DCNT) prevalentes na população brasileira, o presente estudo tem como objetivo analisar fatores potencialmente associados à obesidade abdominal, com ênfase em aspectos sócio-demográficos e comportamentais em uma amostra de usuárias do Sistema Único de Saúde.

\section{MÉ T O D O S}

A coleta de dados foi realizada no Centro Municipal de Saúde (CMS) Carlos Chagas. O centro de saúde selecionado fornece atendimento ambulatorial diário aos residentes da área metropolitana de Belo Horizonte. Trata-se de unidade de saúde localizada em área central de Belo Horizonte com média de 1.600 atendimentos anuais nas principais especialidades médicas. Entre essas, destacam-se pediatria, clínica médica e ginecologia.

Foram selecionadas para o estudo 827 mulheres que atenderam aos seguintes critérios de inclusão: ter idades entre 12 e 65 anos; estar livre de DCNT e residir na área adstrita ao CMS onde foi realizado o estudo. As mulheres foram convidadas a participar do estudo quando eram atendidas nas diversas clínicas do CMS, entre janeiro e julho de 2000. Durante esse período, a equipe da pesquisa freqüentou o CMS diariamente, nos dois turnos de atendimento, para efetivar a captação das participantes.

Para a coleta de dados utilizou-se questionário padronizado sobre questões demográficas, socioeconômicas, comportamentais e história reprodutiva ${ }^{4}$. As medidas de peso e estatura foram realizadas com o indivíduo sem sapatos e sem excesso de roupas ou acessórios. A estatura foi medida com aproximação de $0,1 \mathrm{~cm}$, com o sujeito em posição ereta e a cabeça no plano de Frankfort, utilizando fita métrica inelástica, fixada verticalmente em uma parede. O peso foi medido com uma balança digital (Modelo PL 150, Filizzola S/A, Brasil), com aproximação a $0,1 \mathrm{~kg}$. A circunferência da cintura foi medida com o indivíduo em pé, com o abdômen relaxado. Utilizou-se uma fita métrica de fibra de vidro, no ponto médio entre o rebordo costal e a crista ilíaca. Após uma expiração normal aferiu-se a medida até o milímetro mais próximo. Todas as medidas foram realizadas três vezes por antropometristas previamente treinados, segundo procedimentos padronizados ${ }^{13}$.

A obesidade abdominal, variável dependente do presente estudo, foi definida utilizando-se como ponto de corte valores de circunferência da cintura $\geq 88 \mathrm{~cm}$. Esse ponto de corte tem sido recomendado, por identificar riscos substancialmente aumentados para complicações metabólicas ${ }^{5,6}$.

As seguintes co-variáveis foram consideradas nas análises segundo blocos hierárquicos, definidos de acordo com modelo teórico prévio sobre determinação da obesidade em quatro níveis, a saber (Quadro 1). Bloco 1 (variáveis demográficas e socioeconômicas): faixa etária (12 - 20, 21 - 30, 31 - 40, 41 - 50, 51 - 65 anos), cor de pele (branca, parda, negra), escolaridade $(<8, \geq 8$ anos), renda familiar total em quartis ( $0,0-297,5$; 298,0 - 533,0; 533,5 - 1000,0; 1001,0 - 9000,0 reais), poder aquisitivo segundo a classificação da Associação Brasileira de Pesquisa de Mercado (ABIPEME) (E, D, C, B, A) e estado marital (em união, sem união). A variável cor de pele foi obtida a partir da classificação feita pelo entrevistador. Bloco 2 (variáveis reprodutivas): paridade (nulíparas, $1-4, \geq 5$ partos), idade ao primeiro parto $(<18, \geq 18$ anos), idade de menarca $(<12$, $\geq 12$ anos), menopausa (sim, não) e uso de anticoncepcional hormonal (sim, não). Foram testadas diversas categorizações para a variável paridade, e a apresentada aqui foi a que melhor captou o efeito dessa variável na obesidade abdominal.

As categorias propostas para as variáveis idade ao primeiro parto e idade de menarca estão baseadas em outros estudos desenvolvidos com populações semelhantes ${ }^{14,15}$. A variável faixa etária foi analisada utilizando categorias que representavam décadas, já para a renda, como 
importava ter uma distribuição equilibrada entre categorias, optou-se por trabalhar com quartis. Bloco 3 (variáveis antropométricas): índice de massa corporal $\geq 25,0 \mathrm{~kg} / \mathrm{m}^{2}$ (sim, não) e índice de massa corporal $\geq 30 \mathrm{~kg} / \mathrm{m}^{2}$ (sim, não). Bloco 4 (variáveis comportamentais): tabagismo (sim, não), tempo de tabagismo ( $<10, \geq 10$ anos), consumo de cerveja (diária/semanalmente, quase nunca ou nunca), atividade física no trabalho (leve, moderada, intensa, muito intensa) e atividade física no lazer (leve, moderada, intensa, muito intensa).

As variáveis atividade física no lazer e no trabalho foram obtidas pela aplicação de um questionário validado, que compreende cinco questões referentes a atividade física no trabalho e seis questões referentes a atividade física no lazer $^{16}$. Cada opção de resposta equivale a pontos específicos e os escores são calculados somando-se todos os pontos. As categorias, para ambos os escores, foram definidas levando em consideração a proposta de Shapiro et al. ${ }^{16}$.

As análises estatísticas foram realizadas em etapas. Inicialmente foram calculadas as prevalências de obesidade abdominal e realizadas análises bivariadas, obtendo-se os odds ratio brutos para o conjunto de co-variáveis. A significância das associações bivariadas foi avaliada usando o teste qui-quadrado $\left(\chi^{2}\right)$, qui-quadrado de tendência $\left(\chi^{2}\right)$, Odds Ratio (OR), e intervalos de confiança (IC) de $95 \%$.

A construção do modelo final de regressão logística foi feita segundo proposta de modelos hierarquizados ${ }^{17}$. O modelo teórico de determinação da obesidade abdominal orientou a estruturação dos blocos e a ordem em que as va- riáveis entraram no modelo. As variáveis que apresentaram valor de $p<0,20$ na análise bivariada foram consideradas candidatas para a análise de regressão múltipla. As variáveis dos blocos mais distais (socioeconômico e demográfico) foram as primeiras a entrar no modelo e aquelas que mostraram valor $p<0,20$ foram mantidas no modelo final e serviram de ajuste para variáveis de confusão nos blocos hierarquicamente inferiores. Dessa forma, a razão de odds e valor $p$ apresentados estão ajustados para as variáveis do mesmo nível hierárquico e para os níveis superiores. A associação foi avaliada com o teste da razão de máxima verossimilhança (likelihood-ratio test) e para avaliar a qualidade do ajuste em cada nível hierárquico e do modelo final de regressão logística, utilizou-se o goodness-of-fit test.

As variáveis idade e Índice de Massa Corporal (IMC) foram incluídas no modelo final sob o formato contínuo, com objetivo de controlar confundimento residual. Trata-se de estratégia comumente empregada, que tem como objetivo controlar uma parcela do confundimento não eliminado pelas demais variáveis de controle incluídas no modelo multivariado. O modelo final multivariado não considerou interações, na medida que não foram identificados indícios sobre a presença das mesmas durante o processo de modelagem. Adicionalmente, testou-se para a presença de colinearidade entre as variáveis incluídas no modelo final, não tendo sido, entretanto, identificada a presença.

Os dados foram analisados utilizando-se o software Statistical Package for Social Science (SPSS Incorporation, 2000). Este estudo foi

Quadro 1. Modelo hierárquico de entrada de variáveis no modelo logístico.

\begin{tabular}{|c|c|c|c|c|}
\hline Bloco 1 & Idade & Cor de pele & Renda & Poder aquisitivo \\
\hline Bloco 2 & Paridade & Idade $1^{\circ}$ parto & Idade menarca Menopausa & Uso de anticoncepcionais orais \\
\hline Bloco 3 & IMC & & & \\
\hline \multirow[t]{2}{*}{ Bloco 4} & Tabagismo & Consumo de álcool & Atividade física & \\
\hline & & & Obesidade abdominal & \\
\hline
\end{tabular}

IMC: índice de massa corporal. 
aprovado pelo Comitê de Ética em Pesquisa da Universidade Federal de Minas Gerais (UFMG), e autorizações por escrito foram obtidas de todas as participantes.

\section{RES U L T A D O S}

As mulheres estudadas tinham média de idade de 30 anos, 7,6 anos de escolaridade e 1,7 partos. Somente $7,3 \%$ destas apresentaram atividade física intensa. A prevalência de obesidade abdominal foi de 21,3\% (IC 95\%: 18,6 - 24,3) e de IMC e $\geq 30 \mathrm{~kg} / \mathrm{m}^{2}$ foi de $33,3 \%$ (IC 95\%: $30,1-36,6)$.

Observou-se maior prevalência de obeidade abdominal nas faixas de idades mais avançadas (OR variou entre 2,53 e 23,25). As prevalências de obesidade abdominal também foram maiores entre mulheres de baixa escolaridade (OR=1,76; IC 95\%: 1,25-2,47) e nos grupos de mulheres com níveis de renda mais baixos $(\mathrm{OR}=1,78$ e $\mathrm{OR}=1,83)$ (Tabela 1).

Entre as variáveis reprodutivas, percebe-se aumento na prevalência e potencial chance de desenvolver obesidade abdominal, com o aumento do número de partos (1 - 4 partos, $\mathrm{OR}=2,52$; IC 95\%: 1,65 - 3,85; $\geq 5$ partos, OR=9,63; IC 95\%: 5,23 - 17,72), em mulheres na menopausa (OR=1,70, IC 95\%: 1,00 - 2,86) e naquelas que não usam anticoncepcional $(\mathrm{OR}=1,80$; IC 95\%: 1,14 - 2,85). As duas categorias de IMC analisadas apresentaram associação com a

Tabela 1. Prevalência e Odds ratio (OR) não ajustado para obesidade abdominal, segundo variáveis demográficas e socioeconômicas. Belo Horizonte, 2000.

\begin{tabular}{|c|c|c|c|c|c|}
\hline Variável & $n$ & Prevalência (\%) & OR & IC $95 \%$ & valor- $p^{1}$ \\
\hline \multicolumn{6}{|c|}{ Faixa etária (anos) } \\
\hline $12-20$ & 174 & 6,9 & 1,00 & - & $<0,0001$ * \\
\hline $21-30$ & 273 & 13,6 & 2,13 & $1,03-4,45$ & \\
\hline $31-40$ & 215 & 24,7 & 4,42 & $2,19-9,08$ & \\
\hline $41-50$ & 116 & 37,1 & 7,95 & $3,78-17,00$ & \\
\hline $51-65$ & 49 & 63,3 & 23,25 & $9,50-58,22$ & \\
\hline \multicolumn{6}{|c|}{ Escolaridade (anos) } \\
\hline$<8$ & 407 & 26,0 & 1,76 & $1,25-2,47$ & 0,0011 \\
\hline$\geq 8$ & 420 & 16,7 & 1,00 & - & \\
\hline \multicolumn{6}{|l|}{ Cor da pele } \\
\hline Parda & 460 & 22,6 & 1,18 & $0,81-1,74$ & 0,3797 \\
\hline Negra & 82 & 20,7 & 1,06 & $0,57-1,97$ & 0,8485 \\
\hline Branca & 248 & 19,8 & 1,00 & - & 0,6677 \\
\hline \multicolumn{6}{|l|}{ Poder aquisitivo } \\
\hline A & 26 & 30,8 & 2,15 & $0,67-6,91$ & $0,7732 *$ \\
\hline B & 217 & 21,7 & 1,34 & $0,56-3,22$ & \\
\hline C & 383 & 21,1 & 1,30 & $0,56-3,05$ & \\
\hline D & 160 & 20,6 & 1,26 & $0,51-3,10$ & \\
\hline E & 41 & 17,5 & 1,00 & - & \\
\hline \multicolumn{6}{|c|}{ Renda familiar (Reais) } \\
\hline $0-297,5$ & 203 & 24,1 & 1,78 & $1,07-2,97$ & 0,0254 \\
\hline $298,0-533,0$ & 203 & 24,6 & 1,83 & $1,10-3,05$ & 0,0190 \\
\hline $533,5-1000,0$ & 214 & 22,0 & 1,58 & $0,94-2,63$ & 0,0786 \\
\hline $1001,0-9000,0$ & 192 & 15,1 & 1,00 & & \\
\hline \multicolumn{6}{|l|}{ Estado marital } \\
\hline União & 389 & 23,1 & 1,23 & $0,88-1,72$ & 0,2199 \\
\hline Sem união & 438 & 19,6 & 1,00 & - & \\
\hline
\end{tabular}

${ }^{1}$ Teste de Wald; *Qui-quadrado de tendência. 
obesidade abdominal (Tabela 2). Entre as variáveis comportamentais, percebe-se que a categoria nível de atividade física leve no trabalho apresentou associação significante com a obesidade abdominal (OR=2,91; IC 95\%: 1,26 - 6,73), quando comparada com o nível de atividade física muito intensa (Tabela 3).

Os resultados da análise de regressão logística múltipla identificaram quatro variáveis independentemente associadas à obesidade abdominal: idade (OR variaram entre 2,53 e $27,64)$, escolaridade $<8$ anos (OR=1,49; IC $95 \%$ : 1,02 - 2,18), paridade 1 - 4 filhos (OR=1,80; IC $95 \%$ : $1,04-3,12$ ), e $\geq 5$ partos (OR=3,20; IC 95\%: $1,48-6,90)$ e atividade física leve no trabalho $(\mathrm{OR}=1,63$; IC 95\%: 1,01 - 2,64) (Tabela 4). As variáveis idade e IMC foram incluídas no modelo final no formato contínuo, com o objetivo de controlar confundimento.
D I S C U S S Ã O

O resultado do modelo final de regressão logística hierarquizado revelou que a idade, escolaridade, paridade e a atividade física leve no trabalho permaneceram como variáveis potencialmente associadas à obesidade abdominal, nesta amostra de mulheres usuárias do Sistema Único de Saúde (SUS) e residentes na região metropolitana de Belo Horizonte, Brasil. Estes resultados indicam que essas variáveis têm maior importância no desenvolvimento da obesidade abdominal em relação a outras co-variáveis, como, por exemplo, cor de pele, estado marital, fumo e consumo de álcool. Resultados semelhantes foram descritos por Kac et al. ${ }^{8}$, para amostra probabilística de mulheres residentes no município do Rio de Janeiro, onde a escolaridade influenciou a ocorrência da obesidade abdominal de forma independente e significante. Resultados recentes

Tabela 2. Prevalência e Odds ratio (OR) não ajustado para obesidade abdominal, segundo variáveis reprodutivas e antropométricas. Belo Horizonte, 2000.

\begin{tabular}{|c|c|c|c|c|c|}
\hline Variável & $n$ & Prevalência (\%) & OR & IC $95 \%$ & valor- $p^{1}$ \\
\hline \multicolumn{6}{|c|}{ Paridade (número de partos) } \\
\hline Nulíparas & 296 & 10,8 & 1,00 & - & $<0,0001 *$ \\
\hline $1-4$ & 466 & 23,4 & 2,52 & $1,65-3,85$ & \\
\hline$\geq 5$ & 65 & 53,8 & 9,63 & $5,23-17,72$ & \\
\hline \multicolumn{6}{|c|}{ Menopausa } \\
\hline Sim & 76 & 30,0 & 1,70 & $1,00-2,86$ & 0,0461 \\
\hline Não & 747 & 20,3 & 1,00 & - & \\
\hline \multicolumn{6}{|c|}{ Uso de anticoncepcional } \\
\hline Não & 651 & 23,2 & 1,80 & $1,14-2,85$ & 0,0125 \\
\hline Sim & 174 & 14,4 & 1,00 & - & \\
\hline \multicolumn{6}{|c|}{ Idade ao primeiro parto (anos) } \\
\hline$<18$ & 154 & 27,9 & 0,94 & $0,62-1,43$ & 0,7901 \\
\hline$\geq 18$ & 377 & 26,8 & 1,00 & & \\
\hline \multicolumn{6}{|c|}{ Idade de menarca (anos) } \\
\hline$<12$ & 361 & 21,9 & 1,05 & $0,75-1,47$ & 0,7614 \\
\hline$\geq 12$ & 457 & 21,0 & 1,00 & - & \\
\hline \multicolumn{6}{|c|}{$I M C\left(k g / m^{2}\right)$} \\
\hline$<25,0$ & 773 & 21,0 & 1,00 & $0,70-2,48$ & 0,3899 \\
\hline$\geq 25,0$ & 54 & 25,9 & 1,32 & & \\
\hline \multicolumn{6}{|c|}{$I M C\left(k g / m^{2}\right)$} \\
\hline$<30,0$ & 820 & 21,3 & 0,61 & $0,07-5,13$ & 0,6532 \\
\hline$\geq 30,0$ & 7 & 14,3 & 1,00 & & \\
\hline
\end{tabular}

${ }^{1}$ Teste de Wald; *Qui-quadrado de tendência; IMC: índice de massa corporal. 
Tabela 3. Pevalência e Odds ratio (OR) não ajustado para obesidade abdominal, segundo variáveis de estilo de vida (comportamentais). Belo Horizonte, 2000.

\begin{tabular}{|c|c|c|c|c|c|}
\hline Variável & $\mathrm{n}$ & Prevalência (\%) & OR & IC 95\% & valor- $p^{1}$ \\
\hline \multicolumn{6}{|l|}{ Fumo } \\
\hline Não & 568 & 20,6 & 1,00 & - & 0,7769 \\
\hline Sim & 158 & 22,8 & 1,16 & $0,74-1,74$ & 0,5514 \\
\hline \multicolumn{6}{|c|}{ Tempo que fuma (anos) } \\
\hline Ex-fumante & 101 & 22,8 & 1,16 & $0,68-1,89$ & 0,6209 \\
\hline$<10$ & 74 & 18,9 & 1,00 & - & 0,8734 \\
\hline$\geq 10$ & 84 & 21,4 & 1,17 & $0,53-2,55$ & 0,6036 \\
\hline \multicolumn{6}{|c|}{ Consumo de cerveja } \\
\hline Semanalmente & 95 & 29,3 & 1,18 & $0,68-2,03$ & 0,5551 \\
\hline Quase nunca & 732 & 20,3 & 1,00 & - & \\
\hline \multicolumn{6}{|c|}{ Atividade física no trabalho } \\
\hline Leve & 229 & 27,1 & 2,91 & $1,26-6,73$ & 0,0144 * \\
\hline Moderada & 324 & 21,6 & 2,16 & $0,94-4,95$ & \\
\hline Intensa & 212 & 17,5 & 1,65 & $0,70-3,92$ & \\
\hline Muito intensa & 62 & 11,3 & 1,00 & - & \\
\hline \multicolumn{6}{|c|}{ Atividade física no lazer } \\
\hline Leve & 486 & 21,0 & 1,23 & $0,55-2,72$ & 0,6119 \\
\hline Moderada & 216 & 20,4 & 1,18 & $0,51-2,72$ & 0,6924 \\
\hline Intensa & 80 & 27,5 & 1,75 & $0,71-4,35$ & 0,2252 \\
\hline Muito intensa & 45 & 17,8 & 1,00 & - & 0,5151 \\
\hline
\end{tabular}

${ }^{1}$ Teste de Wald; *Qui-quadrado de tendência.

Tabela 4. Modelo final de regressão logística para obesidade abdominal. Belo Horizonte, 2000.

\begin{tabular}{|c|c|c|c|}
\hline Variável & OR & IC $95 \%$ & valor- $p^{1}$ \\
\hline \multicolumn{4}{|l|}{ Modelo 1} \\
\hline \multicolumn{4}{|l|}{ Faixa etária (anos) } \\
\hline $12-20$ & 1,00 & - & $<0,0001$ * \\
\hline $21-30$ & 2,53 & $1,18-5,44$ & \\
\hline $31-40$ & 5,34 & $2,52-11,30$ & \\
\hline $41-50$ & 8,73 & $4,00-19,02$ & \\
\hline $51-65$ & 27,64 & $11,05-68,69$ & \\
\hline \multicolumn{4}{|l|}{ Escolaridade (anos) } \\
\hline$<8$ & 1,49 & $1,02-2,18$ & 0,0380 \\
\hline$\geq 8$ & 1,00 & & \\
\hline \multicolumn{4}{|l|}{ Modelo 2} \\
\hline \multicolumn{4}{|c|}{ Paridade (número de partos) } \\
\hline Nulíparas & 1,00 & & 0,0113 * \\
\hline $1-4$ & 1,80 & $1,04-3,12$ & \\
\hline$\geq 5$ & 3,20 & $1,48-6,90$ & \\
\hline \multicolumn{4}{|l|}{ Modelo 3} \\
\hline \multicolumn{4}{|c|}{ Atividade física no trabalho } \\
\hline Muito Intensa & 1,00 & - & - \\
\hline Intensa & 1,13 & $0,51-2,50$ & 0,7476 \\
\hline Moderada & 1,02 & $0,59-1,76$ & 0,9394 \\
\hline Leve & 1,63 & $1,01-2,64$ & 0,0459 \\
\hline \multicolumn{4}{|l|}{ Modelo 4} \\
\hline Idade (Contínua) & 1,11 & $1,04-1,18$ & 0,0017 \\
\hline IMC (Contínua) & 0,99 & $0,97-1,01$ & 0,3538 \\
\hline
\end{tabular}

Modelo 1: Idade + Escolaridade; Modelo 2: Modelo 1 + Paridade; Modelo 3: Modelo 2 + Atividade física no trabalho; Modelo 4: Modelo 3 + idade (contínua) + IMC (contínua); ${ }^{\top}$ Teste de Wald; *Qui-quadrado de tendência. 
de um estudo de base populacional, realizado em São Leopoldo, corroboram esses achados ${ }^{9}$.

Observou-se, no presente estudo, maior prevalência de obesidade abdominal em mulheres com cinco ou mais partos. Diversos outros estudos também observaram que mulheres com maior paridade apresentaram maior circunferência da cintura e maior relação cintura quadril $7,8,11$.

Observou-se, no modelo final, associação independente do nível de atividade física de trabalho leve com a obesidade abdominal. Resultados semelhantes também foram descritos por Lanhman et al. ${ }^{11}$ e Rosmond \& Björntorp ${ }^{12}$. A maioria dos obesos é moderadamente ativa e o seu nível de atividade física é limitado pela habilidade em fazer exercícios de alta intensidade. Questões relacionadas ao padrão de atividade física de lazer merecem ser investigadas, levando em consideração a idade, classe social, escolaridade e paridade, pois se supõe que mulheres com melhor poder aquisitivo, maior escolaridade e menor número de partos têm maior preocupação em manter e/ou perder peso. Contudo, um achado que parece apontar na direção oposta a esta hipótese é o resultado do estudo sobre percepção corporal e diagnóstico antropométrico de sobrepeso, realizado em Belo Horizonte, onde se observou que $92,0 \%$ das mulheres não praticavam esporte e apenas 7,6\% faziam caminhadas regulares ${ }^{18}$.

Algumas variáveis perderam poder explicativo na análise múltipla, entre elas o status de menopausa e o uso de anticoncepcionais. Isso pode ter ocorrido porque o uso de anticoncepcional hormonal, por exemplo, pode não indicar, exatamente, a característica reprodutiva em si, mas apenas a condição socioeconômica das poucas mulheres que utilizam esse método de anticoncepção.

Um resultado particularmente surpreendente foi a ausência de associação entre variáveis comportamentais, como o fumo e o consumo de álcool e a obesidade abdominal. Este achado está em desacordo com o estudo de Hofstetter et al. ${ }^{19}$. Em relação ao consumo de álcool, tem sido sugerido que essa variável pode atuar como possível fator de confusão na relação entre fumo e obesidade abdominal. No presente estudo não se verificou associação entre consumo de bebida alcoólica e obesidade abdominal, discordando dos resultados de Lanhman et al. ${ }^{11}$ e Rosmond \& Björntorp ${ }^{12}$, que encontraram associação negativa entre uso de bebida alcoólica e a obesidade abdominal. Machado \& Sichieri20 por outro lado encontraram associação positiva entre a relação cintura-quadril e o consumo de bebida alcoólica em mulheres na menopausa. É possível que tal associação não tenha sido encontrada no presente estudo devido a questões de aferição da variável, que contemplou apenas a freqüência, sem considerar o volume ingerido.

Os resultados apresentados aqui devem ser interpretados com cautela, levando em consideração algumas limitações. Entre essas destacam-se a falta de segurança relacionada à temporalidade de algumas associações mostradas neste estudo e aquelas decorrentes do caráter não probabilístico da amostra.

Em síntese, conclui-se que a idade, a escolaridade, a paridade e a atividade física no trabalho estiveram associadas à obesidade abdominal, no grupo de mulheres aqui estudado, devendo ser sistematicamente consideradas em estudos sobre o tema. Entender a influência que esses fatores exercem na ocorrência da obesidade abdominal é importante para prevenção e promoção da saúde. Assim, como proposta de intervenção, deve-se encorajar modificações no estilo de vida, tais como a prática de exercícios físicos e melhoria nos conhecimentos relacionados aos riscos da obesidade abdominal.

\section{COLABORADORES}

E.O. OLIVEIRA participou na análise de dados e elaboração do manuscrito. G. VELÁSQUEZ-MELÉNDEZ participou na concepção do estudo, coleta e análise de dados e revisão de todas as versões do manuscrito. G. KAC participou na concepção do estudo e revisão de todas as versões do manuscrito. 


\section{RE FER Ê NCIAS}

1. Hidalgo CAG, Kac G, Velásquez-Meléndez G, Valente JG. Factors associated with overweight in Brazilian childbearing-age women according to skin color. Nutr Res. 2002; 22(7):785-94.

2. Kain J, Vio F, Albala C. Obesity trends and determinant factors in Latin America. Cad Saúde Pública. 2003; 19(Suppl 1):S77-S86.

3. Molarius A, Seidell JC. Selection of anthropometric indicators for classification of abdominal fatness: a critical review. Int J Obesity. 1998; 22(8):719-27.

4. Velásquez-Meléndez G, Kac G, Valente JG, Tavares R, Garcia ES. Evaluation of waist circumference to predict general obesity and arterial hypertension in women in Greater Metropolitan Belo Horizonte, Brazil. Cad Saúde Pública. 2002; 18(3):765-71.

5. Han TS, van Leer EM, Seidell CJ, Lean MEJ. Waist circumference action levels in the identification of cardiovascular risk factors: prevalence study in a random sample. BMJ. 1995; 311(7017):1401-5.

6. Scarcela C, Despres JP. Tratamiento de la obesidad: necesidad de centrar la atención en los pacientes de alto riesgo caracterizados por la obesidad abdominal. Cad Saúde Pública. 2003; 19(Supl 1): S7-S19.

7. Castanheira M, Olinto MTA, Gigante DP. Associação de variáveis sócio-demográficas e comportamentais com a gordura abdominal em adultos: estudo de base populacional no Sul do Brasil. Cad Saúde Pública. 2003; 19(Supl 1):S55-S65.

8. Kac G, Velásquez-Meléndez G, Coelho MASC. Fatores associados à obesidade abdominal em mulheres em idade reprodutiva. Rev Saúde Pública. 2001; 35(1):46-51.

9. Olinto MT, Nacul LC, Gigante DP, Costa JS, Menezes AM, Macedo S. Waist circumference as a determinant of hypertension and diabetes in Brazilian women: a population-based study. Public Health Nutr. 2004; 7(10):629-35.

10. Olinto MT, Nacul LC, Dias-da-Costa JS, Gigante DP, Menezes AM, Macedo S. Níveis de intervenção para obesidade abdominal: prevalência e fatores associados. Cad Saúde Pública. 2006; 22(6): 1207-15.
11. Lahmann PH, Lissner L, Gullberg B, Berglund G. Sociodemographic factors associated with long-term weight gain, current body fatness and central adiposity in Swedish women. Int J Obes Related Metab Disord. 2000; 24(6):685-94.

12. Rosmond R, Björntorp P. Psychosocial and socio-economic factors in women and their relationship to obesity and regional body fat distribution. Int J Obesity. 1999; 23(2):138-45.

13. Lohman TG, Roche AF, Martorell R. Anthropometric standardization reference Manual. Champaign: Human Kinetics Books; 1988.

14. Kac G, Benício MHDA, Velásquez-Meléndez G, Valente JG. Postpartum weight retention among women in Rio de Janeiro: a follow-up study. Cad Saúde Pública. 2003; 19(Suppl 1):149S-61S.

15. Kac G, Benício MHDA, Velásquez-Meléndez G, Valente JG. Nine months postpartum weight retention predictors for Brazilian women. Public Health Nutr. 2004; 7(10):661-8.

16. Shapiro S, Weinblatt E, Frank CW, Sager RV. The H.I.P. study of incidence and prognosis of coronary heart disease; preliminary findings on incidence of myocardial infarction and angina. J Chron Dis. 1965; 18(6):527-58.

17. Victora CG, Huttly SR, Fuchs SC, Olinto MTA. The role of conceptual frameworks in epidemiological analysis: a hierarchical approach. Int J Epidemiol. 1997; 26(1):224-7.

18. Marçal A, Coelho S, Marçal KA, Kac G, VelásquezMeléndez G. Avaliação da concordância entre a percepção do peso corporal e o diagnóstico antropométrico de sobrepeso em mulheres atendidas em um centro de saúde de Belo Horizonte. Rev Mineira Enfermagem. 2001; 5(1):7-12.

19. Hofstetter A, Schutz $Y$, Jéquier $E$, Wahren J. Increased 24-hour energy expenditure in cigarette smokers. N Eng J Med. 1986; 314(2):79-82.

20. Machado PAN, Sichieri R. Relação cintura-quadril e fatores de dieta em adultos. Rev Saúde Pública. 2002; 36(2):198-204.

Recebido em: 3/3/2006

Versão final apresentada em: 18/4/2007 Aprovado em: 11/5/2007 